\title{
The Analysis of Bamboo Sheet Mechanical Properties in the Process of Shaping and Study on Application
}

\author{
Jian Yang ${ }^{1, ~ a}$, Sheng Jia ${ }^{2, b}$, Yixiang Peng ${ }^{3, c}$, and Yanfang Wang ${ }^{4, d}$ \\ College of Nuclear Technology and Automation Engineering, Chengdu University of Technology, \\ Sichuan, 610059 , China \\ ayangjian@cdut.edu.cn; 'b1064531383@qq.com; '315456331@qq.com; d810623788@qq.com
}

Keywords: Bamboo sheet, finite element analysis, mechanical model, revolving tongs.

\begin{abstract}
Detailed studies about the deformation principles and processing mechanisms of bamboo sheet cell cube are theoretical foundation to realize mechanization, automation and informatization of bamboo products manufacturing, and also the key to develop different kinds of bamboo knit machine. For internal stress and external torques needed here are changing constantly during all the time of bamboo sheet's bending and ring up, this paper use ANSYS software to establish a finite element analysis model for a piece of bamboo sheet which is in $450 \mathrm{~mm}$ length, $16 \mathrm{~mm}$ wide and $1 \mathrm{~mm}$ thickness, and study its mechanical properties under different torques. The model shows the stress distribution and bamboo deformation regular patterns, which reveals the relations between revolving control positions and the needed moments of the two pairs of revolving tongs. Based on these studies, a pair of adjustable revolving tongs applying on a special bamboo shaping machine which can automatically shape 20 pieces of bamboo sheets simultaneously without slipping was innovative developed.
\end{abstract}

\section{Introduction}

China, the richest country in bamboo resource, has become a giant in bamboo product producing and exporting area. But problems, such as fast increasing of labor cost, lacking of high-technology product, poor innovation ability of enterprise and other issues like serious homogenization etc., have become increasingly prominent[1][2]. Using the fabrication process innovation of bamboo to enhance product contribution value and promote mechanization, automation and informatization of processing are the keys to solve the problems at present. The researches of new bamboo products, which made up of the unit body of bamboo sheet, mat and curtain, make the study on relevant basic theories of bamboo processing and the application in the field of mechanical automatic processing equipment more and more urgent and important.

At present, domestic investigations about the structure of bamboo is much more than foreign countries. Zhang Yamei, a member of Research Institute of Industry, Chinese Academy of Forestry, studied the mechanical properties of moso bamboo under thermal treatment [3]. It showed that the modulus of rupture of moso bamboo goes up with time at first, and then down after $100^{\circ} \mathrm{C}$ to $200^{\circ} \mathrm{C}$ thermal treatment. However, the impact on modulus is not obvious. This study has a significant influence on the processing of shaping bamboo in high temperature. Zhang Peili of Nanjing University of Aeronautics and Astronautics, adopted microscope and picture processing technology to study the vascular bundle spatial distribution of moso bamboo and set up a bridge between bamboo microstructure and mechanical property, obtaining kinds of parameters about bamboo micro dynamics model[4]. Yang Yunfang conducted a large number of experiments about bamboo patterns, getting many elastic modulus and tensile strength data[5]. Xian Dingguo has detailed researches about the influence of different microstructure to the mechanical properties [6]. Grosser D. and Liese Wstudied 52 species of bamboo from 7 Asian counties, and classified vascular bundle [7]. Shigeysu Amada has studied the characters of bamboo including mechanical properties and temperature reaction [8].

Above researches mostly concentrate on theoretical counting, bamboo biology character and mechanical properties. While dealing with thin bamboo sheet, this paper focus on studying its 
mechanical properties under different torques, adopting finite element analysis method to get the stress distribution and bamboo deformation regular patterns, which lays the theoretical foundation of the preparation processing and knit technology for bamboo materials. Based on that, a new type of automatic bamboo sheets frame shaping machine was designed for the first time. This machine, using adjustable involving tongs, can accomplish 20 pieces of bamboo sheets shaping, heating, and fixating once a time automatically. It solves problems of bamboo sheet frame shaping, such as lowefficiency, concentrated-labor, and low-safety, etc.

\section{Finite Element Analysis}

Model Information. Bamboo is a kind of nature organic macromolecule polymer, composed of vascular bundles and parenchyma cells. Vascular bundle possesses good rigidity, besides parenchyma cells live among them working as a buffer when sustains force, bamboo sheet shows good elasticity. However in practice, the mechanical properties of bamboo are not stable and it's hard to forecast by theoretical methods, so the analysis of bamboo has become a conundrum. Macroscopic mechanical properties of bamboo appearing a big dispersal are due to the number of vascular bundles varying with the height of node. This character is not beneficial for forecasting its strength in processing. This research uses ANSYS to simulate the process of a piece of bamboo sheet bending from a line to circle. The model meets the following assumptions:1)Vascular bundle and matrix are materials of isotropy.2)Vascular bundle and matrix are constant and the proportion is settled.3)The cell cube of model is SOLID45, whose every node has X、Y、Z three displacement degrees of freedom.

Bending Simulation. The process of shaping the bamboo sheet is divided into 3 steps. Firstly, make a sheet circle by opposite directions face to face moving and the revolving of two pairs of tongs. And then, glue it. Thirdly, shape the circle into wanted shape by 4 to 6 heat sticks. It's very important for establishing the model to study the positions of revolving tongsand the torque should be offered by the motors.

Setting Constraints and Torques. The model is a rectangle sheet in $450 \mathrm{~mm}$ length, $16 \mathrm{~mm}$ wide and $1 \mathrm{~mm}$ thickness as shown in Figure 1. A pair of revolving tongs clamps A point, and another is B. Taking tongs working area into account, the pressure, acting on $16 \mathrm{~mm}^{2}$ area and changing with time, is put on the two ends of sheet. In the process of bending, A and B go in the opposite directions face to face at the same uniform speed. A moves to D, B moves to C.

Simulation Analysis. Existed researches indicate that the tensile modulus of elasticity parallel to grain of moso bamboo vascular bundle is $27.6 \mathrm{Gpa}$ and that of matrix is $6.1 \mathrm{Gpa}$. Considering the universality of machine in processing sheets of different kinds of bamboo or with different height of node, chooses a sheet whose proportion of vascular bundle is $50 \%$ to get the tensile modulus of elasticity parallel to grain and the result is $15 \mathrm{Gpa}$. Poisson ratio decides transverse stain but the area of bamboo sheet won't shrink much when it's bended, so the Poisson ratio has little effect on mechanical characteristic of bamboo and take an average value 0.28 . According to above data, add a constant rising torque to simulate a motor when bending the sheet to get stress distribution and deformation regular patterns. As shown in Figure 2 , the torque needed for different degree of bending will be got.

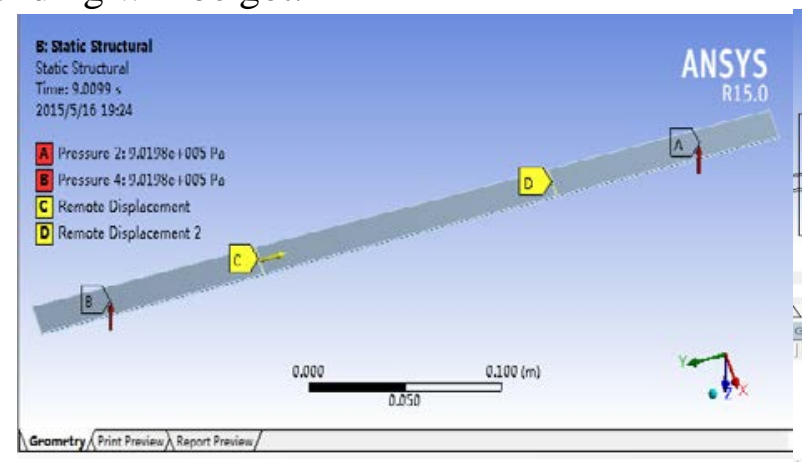

Fig. 1 model setting

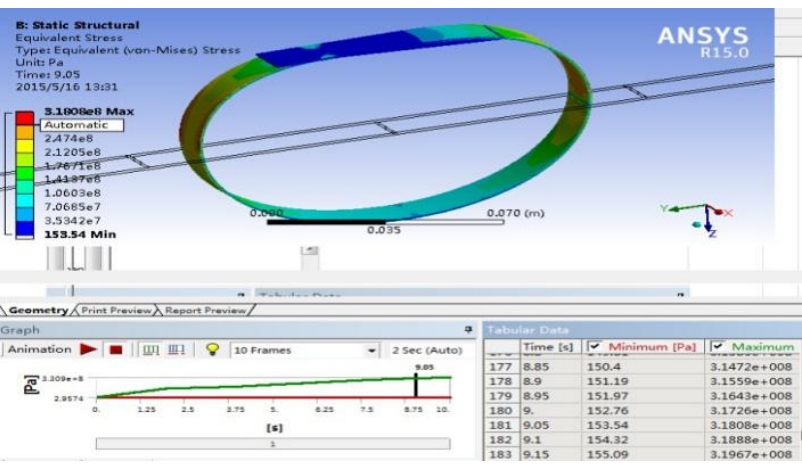

Fig. 2 result of analysis 
The ANSYS simulation shows that the time to approach the expected bending position is 9 seconds and the torque is $1.152 \mathrm{~N} \cdot \mathrm{m}$ at that moment. Having experiments on an experimental prototype for a piece of bamboo sheet, the torque is almost the same as simulation result from which reveals the relations between the shape of bamboo sheet and the two pairs of tongs' needed torque, and this is used as a gist to choose motors of revolving tongs and design a control system.

whereas the distances of clamping points in moving influence the real stress distribution and deformation regular patterns a lot, take two ways to simulate a piece of bamboo sheet deformation in order to study such a phenomenon.

Method I : Clamping points do not move with a way of translation. The largest deformation under pressure of $18 \times 10^{5}$ pa is shown as Figure 3 .

Method II : Clamping points move with a way of translation. The biggest deformation under pressure of $9 \times 10^{5}$ pa is shown as Figure 4 .

Compared method II to I, the deflection is dispersed, in another words, the deformation is spread to the whole sheet. What's more, the needed torque to approach the same deformation is half of I and the biggest part stress decrease to $27 \%$. The result of simulation indicates that the bigger dispersion of deflection is, the stain will be smaller and the needed torque of motor is smaller. In the control of shaping process, the revolving torque of tongs and part stress will be effectively cut down, when make the sheet revolve after approaching.

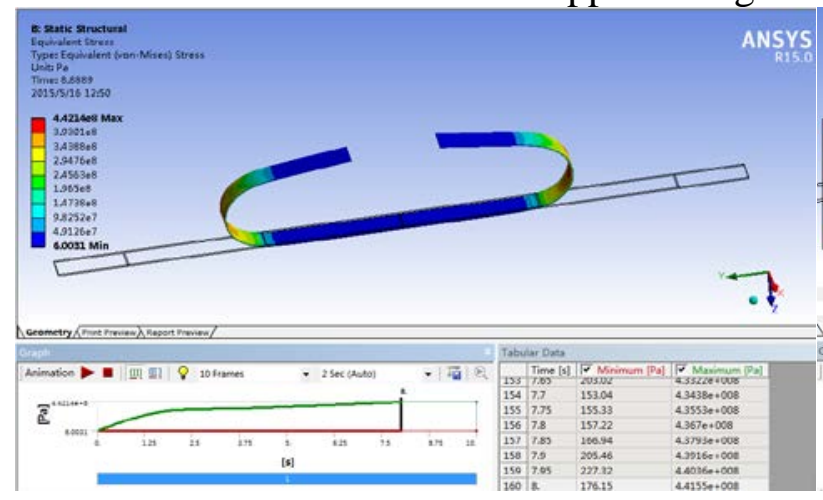

Fig.3 the result of method I

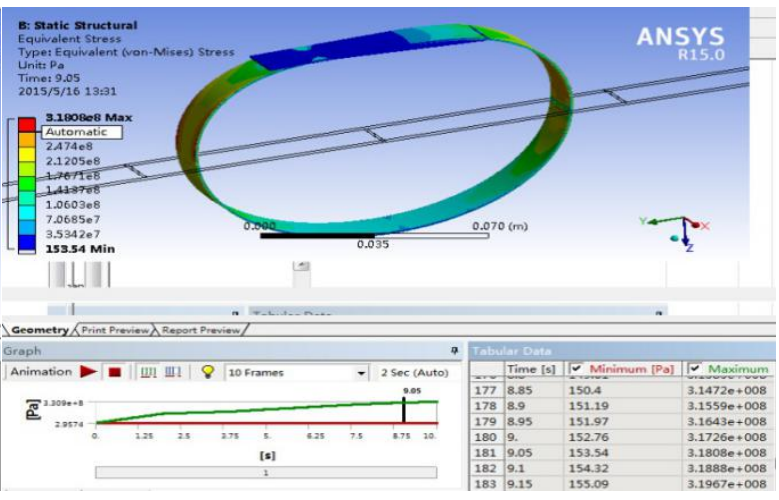

Fig.4 the result of method II

Test of Experiment. This conclusion was proved in the test of an experimental prototype. In the test, the type of motor is BS42HB60-01(parallel connection) and its keeping torque is $0.8 \mathrm{~N} \cdot \mathrm{m}$. Because the torque of step motor is constant, its power will rise up with rotational speed but it's not always linear. When the speed reaches to 200rpm, the torque will decline. Therefore, the torque of motor could be regarded as constant $0.8 \mathrm{~N} \cdot \mathrm{m}$ under the speed of 200rpm. In simulation analysis, the proportion of vascular bundle is $50 \%$, but it's $30 \%$ in the practical processing and experimental prototype test, and thus it's safe and common for the choice of elastic modulus in simulation. For the test, 11 pieces of bamboo sheets whose length varied from $400 \mathrm{~mm}$ to $2400 \mathrm{~mm}$ were tested and a pair of tongs was driven by one motor. Taking method I to have tests, all toques are over $0.8 \mathrm{~N} \cdot \mathrm{m}$ and motors are out of step. In method II, the start distance of two clamping points is 2/3 of sheet's length, and the tongs begin to revolve when the opposite directions distance of two pair of tongs reaches to $1 / 3$ of sheet's length, the motors are not out of step.

\section{Revolving tongs design.}

There is relative slipping tendency between the sheet and the tongs in the process of shaping under the action of the self-gravity and elasticity of sheet. If the force of friction is too small to stop the relative slipping, the precision will reduce. Since the machine should be qualified to different batch production, a sort of adjustable compression tongs was designed based on the simulation outcome. The up and down splints are made of aluminum and the top and bottom surfaces of splints are attached silicone sponge whose compressing degree is controlled by the two sides screw bolts and nuts. 


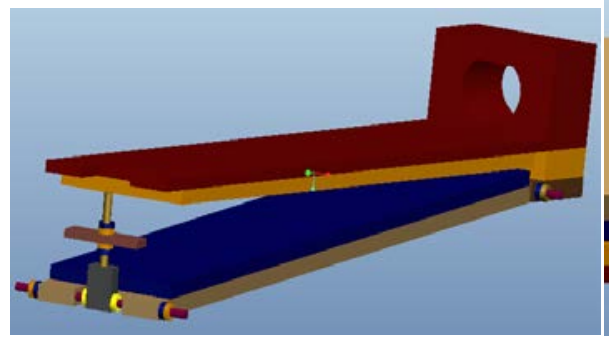

(a)

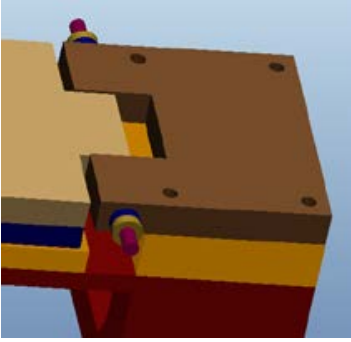

(b)

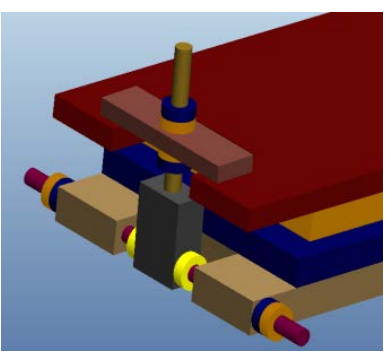

(c)

Figure 5 Adjustable compression tongs

At the beginning of shaping, the tongs are in horizontal direction. The maximum numbers of sheets, which tongs could hold is 20 . When shape, the existed uncertain factor may lead the middle part of sheet bending up or down in the opposite direction moving. For example, when tongs revolve at the program setting point, the two ends of sheet may intervene with bending up part, resulting in failing of revolving. To solve this problem, control system should be designed to let the relative center of two pairs of tongs generate some skewing at the start of shaping. But this also have demands to structure design of tongs. At first, the central line of tongs cannot go across the axle centre of rotation axis, if not, no matter how tongs revolve, the sheet will be dragged by the tongs and the force of friction among silicone sponge is much less than the tensile stress, that will make the sheet slip. In consideration of that, rotation axis and central line must have a vertical distance shown in Figure 6 and Figure 7. In order to ensure the centre point of sheet moving down, the two pairs of tongs should revolve in opposite directions back to back for tongs' position under rotation axis, on the contrary, should do in opposite directions face to face. The former in Figure 6 will pull the sheet, but latter will shrink in Figure 7. It has concluded that pull will make the sheet slip, but shrink will make the sheet between two clamping points change from line to arc and the displacement of every point relative to clamping points is downward because of the good elasticity and thin thickness of bamboo, so that won't slip.

\section{Conclusion.}

Deeply studying the bamboo deformation and process mechanism of bamboo sheet cell cube is not only a theoretical foundation of realizing mechanization, automation and informatization of bambooproduct manufacturing, but also the key to develop different kinds of bamboo sheet knit machine. The data of physical mechanics performance applied in infinite element analysis model is bigger than that of references to assure the reliability and commonality of simulation conclusion. The regular patterns of stress distribution and bamboo deformation as well as the relationship between different shape and needed torque of the two pairs of revolving tongs can be references for developing new types of bamboo products or automatically manufacturing machine.

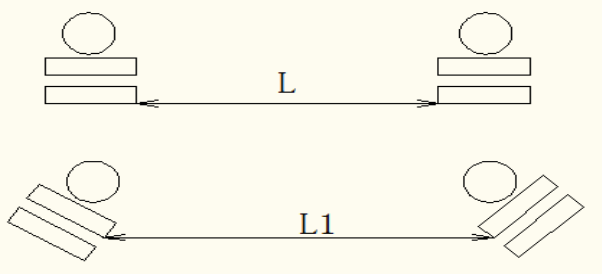

Fig. 6 under rotation axis

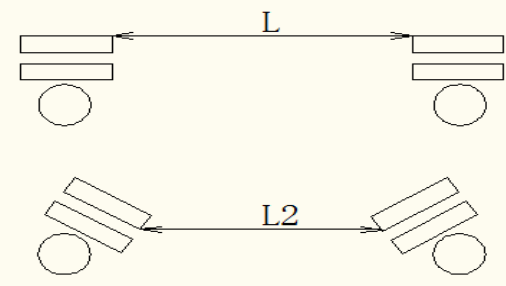

Fig. 7 above rotation axis

\section{Acknowledgments.}

The research work developed here was partly supported by Chengdu University of Technology mechanical engineering teaching innovation team project (10912-JXTD201501) and National Undergraduate Training Program for Innovation and Entrepreneurship (Bamboo sheets frame shaping machine). We acknowledge the work of all those who contributed to earlier versions of the paper. 


\section{References}

[1] LI Yanjun. XU Bin. ZHANG Qisheng. JIANG Shenxue.Present situation and the countermeasure analysis of bamboo timber processing industry in China[J].Journal of Forestry Engineering., 2016,1( 1) :2-7.

[2]XING Li-ping. BAO Yu-ying. MA Yan. REN Hong-e. YANG Chun-mei.Technolgy and Equipment for all Sides Milling and Three Sides Splitting of Bamboo [J].Journal of West China Forestry .2014,43(4),66-70.

[3]Zhang Ya-mei. YU Yang-lun. YU Wen-ji.Effect of heat treatment on mechanical properties of phyllostachys pubescens bamboo[J].Transactions of Materials and Heat Treatment.2009,30-35.

[4]Zhang Pei-duan.Micromechanical model and finite element analysis of bamboo[J].Nanjing University of Aeronautics and Astronautics.2011.

[5]Yang Yunfang(Zhejiang Forestry College,Lin'an 311300,PRC)and Liu Zhikun .Phyllostachys Pubescens Wood :Tensile Elastic modulus and Tensile Strength[J].Zhejiang For Coll, 1996,13 (1), 21-27.

[6] Xiau Xingjuan. Xian Dingguo.The relationship of Microstructture and mechanical properties of bamboo[J].Journal of Bamboo Research.1996, 11-23.

[7] Grosser D. Liese W. On the anatomy of Asian bamboos, with special reference to their vascular bundles[J]. Wood Science and Technology.1971(5),290-312.

[8] Shigeysu Amada. Sun Untao.Fracture Properties of Bamboo[J].Composites: Part B,2001(43) ,21-26. 